\title{
The easier, the better: Age, creatinine, ejection fraction score for operative mortality risk stratification in a series of 29,659 patients undergoing elective cardiac surgery
}

\author{
Marco Ranucci, MD, ${ }^{\mathrm{a}}$ Serenella Castelvecchio, MD, ${ }^{\mathrm{a}}$ Massimiliano Conte, $\mathrm{MD},{ }^{\mathrm{b}}$ Gianluigi Megliola, MD, \\ Giuseppe Speziale, MD ${ }^{\mathrm{c}}$ Flavio Fiore, MD, ${ }^{\mathrm{c}}$ Fabio Guarracino, MD ${ }^{\mathrm{d}}$ Sabino Scolletta, MD, \\ Ricardo Martinez Escobar, MD, ${ }^{\mathrm{f}}$ Mauro Falco, MD, ${ }^{\mathrm{g}}$ Elena Bignami, MD, ${ }^{\mathrm{c}, \mathrm{h}}$ and Giovanni Landoni, $\mathrm{MD}^{\mathrm{h}}$
}

Objective: Age, preoperative creatinine value, and ejection fraction are easily arranged in the age, creatinine, ejection fraction score to predict operative mortality in elective cardiac operations, as recently shown. We validate the age, creatinine, ejection fraction score in a large multicentric study.

Methods: We analyzed 29,659 consecutive patients who underwent elective cardiac operations in 14 Italian institutions during the period from 2004 to 2009 . The operative (30-day) mortality rate was recorded for the entire population and for subgroups of patients based on the risk distribution. The predicted mortality was assessed using the additive and logistic European System for Cardiac Operative Risk Evaluations, and the age, creatinine, ejection fraction score. Accuracy and clinical performance of the different models were tested.

\begin{abstract}
Results: The observed mortality rate was $2.77 \%$ ( $95 \%$ confidence interval, $2.59-2.96)$. The predicted mortality rate was $2.84 \%$ (95\% confidence interval, $2.79-2.88$ ) for the age, creatinine, ejection fraction score (not significantly different from the observed rate), 6.26\% for the additive European System for Cardiac Operative Risk Evaluation, and 9.67\% for the logistic European System for Cardiac Operative Risk Evaluation (both significantly overestimated). For all deciles of risk distribution, the European System for Cardiac Operative Risk Evaluation significantly overestimated mortality risk; the age, creatinine, ejection fraction score slightly overestimated the mortality risk in very low-risk patients and significantly underestimated the mortality risk in very high-risk patients, correctly estimating the risk in 7 of 10 deciles. The accuracy of the age, creatinine, ejection fraction score was acceptable (area under the curve of 0.702). In a separate analysis, this value increased to 0.74 by excluding centers that reported no operative mortality. These values were similar or worse for the European System for Cardiac Operative Risk Evaluation.
\end{abstract}

Conclusions: The age, creatinine, ejection fraction score provides an accuracy level comparable to that of the European System for Cardiac Operative Risk Evaluation, with far superior clinical performance. (J Thorac Cardiovasc Surg 2011;142:581-6)

Earn CME credits at

http://cme.ctsnetjournals.org

The number of patients who undergo cardiac operations worldwide currently exceeds 1 million annually. This

\footnotetext{
From the IRCCS Policlinico San Donato, ${ }^{a}$ Milan, Italy; Città di Lecce Hospital-GVM Care and Research, ${ }^{\mathrm{b}}$ Lecce, Italy; Anthea Hospital-GVM Care and Research, ${ }^{\mathrm{c}}$ Bari, Italy; the Azienda Ospedaliera Universitaria Pisana, ${ }^{\mathrm{d}}$ Pisa, Italy; Policlinico Le Scotte, University of Siena, ${ }^{e}$ Italy; Fondazione Poliambulanza Istituto Ospedaliero, ${ }^{\mathrm{f}}$ Brescia, Italy; European Hospital, ${ }^{\mathrm{g}}$ Rome, Italy; and Università Vita-Salute San Raffaele, ${ }^{\text {h }}$ Milan, Italy.

Disclosures: Authors have nothing to disclose with regard to commercial support.

Received for publication June 8, 2010; revisions received Sept 20, 2010; accepted for publication Nov 19, 2010; available ahead of print June 27, 2011.

Address for reprints: Giovanni Landoni, MD, Department of Cardiothoracic Anesthesia and Intensive Care, Istituto Scientifico San Raffaele, Via Olgettina 60, Milano, 20132 Italy (E-mail: landoni.giovanni@hsr.it).

$0022-5223 / \$ 36.00$

Copyright (c) 2011 by The American Association for Thoracic Surgery doi:10.1016/j.jtcvs.2010.11.064
}

surgery is burdened by a considerable use of financial and human resources, and the problem of mortality risk stratification is becoming increasingly important.

The age, creatinine, ejection fraction (ACEF) risk score for operative mortality in patients undergoing elective cardiac surgery was proposed by Ranucci and coworkers ${ }^{1}$ in 2009. This score is based on 3 factors only: age, preoperative creatinine value, and ejection fraction (EF). The main characteristic of this score is the use of age and EF as continuous variables; the ratio between age (years) and $\mathrm{EF}(\%)$ constitutes the basic calculation; an additional point is added if the preoperative creatinine value exceeds $2.0 \mathrm{mg} /$ $\mathrm{dL}$. The ACEF score was developed and validated in patients undergoing elective cardiac surgery (excluding patients with congenital heart disease) in a single institution. When compared with other existing risk scores (additive and logistic euroSCOREs, Parsonnet, Northern New England, Cleveland Clinic), the ACEF score provides comparable or better accuracy values, with improved calibration and clinical performance. ${ }^{1}$ The ACEF score was recently ${ }^{2}$ 


Abbreviations and Acronyms
$\begin{array}{ll}\text { ACEF } & =\text { age, creatinine, ejection fraction } \\ \text { AUC } & =\text { area under the curve } \\ \text { CI } & =\text { confidence interval } \\ \text { EF } & =\text { ejection fraction } \\ \text { euroSCORE } & =\text { European System for Cardiac } \\ & \text { Operative Risk Evaluation } \\ \text { PROM } & =\text { predicted risk of mortality } \\ \text { STS } & =\text { Society of Thoracic Surgeons }\end{array}$

incorporated into the Syntax score, and the resulting clinical Syntax score was more accurate in predicting outcome after coronary angioplasty.

Until now, no external validation studies comparing the ACEF score with the euroSCORE have been published. The present study aims to validate the ACEF score and to compare it with the euroSCORE in terms of accuracy and clinical performance in a large series of Italian patients who underwent elective cardiac surgery in 14 Italian institutions between 2004 and 2009.

\section{MATERIALS AND METHODS}

Fourteen Italian institutions contributed to this study: IRCCS Policlinico S. Donato, Milan; Università Vita-Salute San Raffaele, Milan; Anthea Hospital, Bari; Città di Lecce Hospital, Lecce; Villa Maria Cecilia Hospital, Cotignola; Villa Azzurra Hospital, Rapallo; Villa Maria Pia Hospital, Torino; Villa Maria Eleonora Hospital, Palermo; Villa Maria Beatrice Hospital, Firenze; Salus Hospital, Reggio Emilia; Policlinico Le Scotte, Siena; Azienda Ospedaliera Universitaria Pisana, Pisa; Fondazione Poliambulanza Istituto Ospedaliero, Brescia; and European Hospital, Rome.

The study was approved by the local ethics committee or the institutional review board of the participating institutions; given the retrospective nature of data collection based on existing databases, the need for an informed consent was waived.

Data were prospectively collected in local databases at each institution since 2004. The start date of data collection differed from institution to institution. All the institutions contributed at least 300 cases per year. Data collected at the IRCCS Policlinico S. Donato included only patients who underwent operation after October 2008. Data from the GVM hospitals (Anthea Hospital, Bari; Città di Lecce Hospital, Lecce; Villa Maria Cecilia Hospital, Cotignola; Villa Azzurra Hospital, Rapallo; Villa Maria Pia Hospital, Torino; Villa Maria Eleonora Hospital, Palermo; Villa Maria Beatrice Hospital, Firenze; and Salus Hospital, Reggio Emilia) were collected in a single, centralized database. These parameters excluded the patient population used to develop and internally validate the ACEF score in the first study. ${ }^{1}$ All the institutions routinely and prospectively collected patient data and stratified the operative mortality risk using the additive ${ }^{3}$ and logis$\mathrm{tic}^{4}$ euroSCOREs. The information needed to calculate the euroSCORE was collected routinely as part of clinical care and follow-up by surgeons, anesthesiologists, and intensivists, according to the local policies. Operative mortality information was obtained by telephone contact with the patient and family members, and a medical practitioner. Operative mortality was defined according to the Society of Thoracic Surgeons (STS) definition, as mortality including (1) all deaths occurring during the hospitalization in which the operation was performed, even if after 30 days; and (2) those deaths occurring after discharge from the hospital, but within 30 days of the procedure unless the cause of death is clearly unrelated to the operation.
Among the fields used to calculate the euroSCORE, age, preoperative creatinine value, and left ventricular EF were available to calculate the ACEF score.

Each institution contributed to the general data collection with a minimum of 307 to a maximum of 6432 cases. All the cases were collected consecutively at each institution.

Patients were excluded if they were aged less than 18 years, had congenital heart disease, were nonelective (euroSCORE fields: critical preoperative state, emergency, surgery of acute ventricular septal defect), or were undergoing cardiac transplantation.

The initial dataset comprised 30,231 patients. Follow-up was completed in $98.1 \%$ (range $96.9 \%-99.3 \%$ per institution) of the patients discharged from the hospital, leading to a total of 29,659 patients. Data were collected and sent to the collecting institution (Università Vita-Salute San Raffaele) in an Excel (Microsoft Corporation, Redmond, Wash) format, including age, preoperative serum creatinine value, EF, additive and logistic euroSCOREs, and operative mortality. Each institution received a code number at the collecting institution. Data were subsequently sent to the analyzing institution (San Donato Hospital) with the blinded code number.

At the analyzing institution, all data were merged into a single Excel file and subsequently converted into an SPSS 13.0 (SPSS Inc, Chicago, Ill) computerized statistical program that was used for all the statistical calculations.

The ACEF score was calculated according to the equation ${ }^{1}$

ACEF score $=$ age $(\mathrm{y}) / \mathrm{EF}(\%)$

+1 (if preoperative serum creatinine value $>2.0 \mathrm{mg} / \mathrm{dL}$ ).

The euroSCORE provides a direct evaluation of the operative mortality risk; the operative mortality risk according to the ACEF score was calculated according to the logistic equation ${ }^{1}$

$$
\begin{aligned}
\text { operative mortality risk }(\%)= & 100 \times\left(\exp ^{-5.4+1.24} \times \mathrm{ACEF}\right) / \\
& \left(1+\left[\exp ^{-5.4+1.24} \times{ }^{\mathrm{ACEF}}\right]\right) .
\end{aligned}
$$

Model discrimination (accuracy) of the 3 predictive models was assessed using a receiver operating characteristic analysis, producing an area under the curve (AUC) with $95 \%$ confidence intervals (CIs). The same analysis was repeated for the entire patient population and for subgroups of lowrisk (additive euroSCORE $\leq 2$ ), medium-risk (additive euroSCORE $3-5$ ), and high-risk (additive euroSCORE $>5$ ) patients.

Calibration and applicability (clinical performance) of the model were assessed by comparing the observed mortality rate ( $\%$ with $95 \% \mathrm{CI})$ with the predicted mortality rate ( $\%$ with $95 \% \mathrm{CI}$ ) for each of the 3 models. The analysis was repeated for the entire patient population and for subgroups of patients stratified to deciles according to the logistic euroSCORE-predicted mortality.

\section{RESULTS}

Table 1 shows the characteristics of the patient population. A total of 2956 patients $(10 \%)$ were high risk (additive euroSCORE > 10), with $2220(7.5 \%)$ aged more than 80 years, $1270(4.2 \%)$ with an EF less than 0.3 , and 1003 $(3.4 \%)$ with preoperative serum creatinine values greater than $2.0 \mathrm{mg} / \mathrm{dL}$.

A preliminary test of the linearity assumption for the relationship age/EF versus mortality was performed, and the hypothesis was confirmed with an $r^{2}$ coefficient of 0.94 at the linear regression between deciles of age/EF and mortality rate.

The observed mortality rate was $2.77 \%$. The mortality rate predicted by the ACEF score was $2.84 \%$, which was 
TABLE 1. Characteristics of patient population $(N=29,659)$

\begin{tabular}{lcccc}
\hline \multicolumn{1}{c}{ Variable } & Mean & SD & 95\% CI & Range \\
\hline Age (y) & 66.6 & 11.6 & $66.5-66.7$ & $19-93$ \\
Serum creatinine (mg/dL) & 1.05 & 0.57 & $1.04-1.06$ & $0.2-12$ \\
Left ventricle EF (\%) & 54.3 & 9.75 & $54.2-54.4$ & $10-86$ \\
Additive euroSCORE & 6.26 & 3.2 & $6.22-6.3$ & $0-33$ \\
Logistic euroSCORE & 9.67 & 10 & $9.56-9.79$ & $0.8-99$ \\
ACEF score & 1.32 & 0.48 & $1.32-1.33$ & $0.17-7.3$ \\
ACEF score-predicted mortality $(\%)$ & 2.84 & 3.66 & $2.79-2.88$ & $0.5-97$ \\
Observed mortality (\%) & 2.77 & 0.16 & $2.59-2.96$ & - \\
\hline
\end{tabular}

SD, Standard deviation.

not significantly different from the observed mortality rate. The mortality rates predicted by the additive euroSCORE $(6.26 \%)$ and the logistic euroSCORE $(9.67 \%)$ were significantly $(P=.001)$ overestimated in comparison with the observed mortality risk.

Receiver operating characteristic analysis (Figure 1) demonstrated similar values of accuracy. The AUC was similar in the 3 risk scores, 0.702 for the ACEF score, 0.692 for the logistic euroSCORE, and 0.687 for the additive euroSCORE, with overlapping $95 \%$ CIs. The odds ratio for the ACEF score was 2.58 (95\% CI, 2.35-2.83), with an estimated operative mortality increase of $94 \%$ per each ACEF score point.

To better assess the accuracy of the ACEF score and the euroSCORE in different institutions, a further institutionbased analysis was conducted (Table 2). The institutions coded 1 and 2 provided a dataset with $0 \%$ operative mortality. Therefore, they could not be assessed for accuracy of the models. In this analysis, data from the GVM hospitals are pooled together because they belong to the same central database. The ACEF score demonstrated AUC values of 0.74 or more in 4 of the 5 institutions. There was a total lack of accuracy of all models in 1 institution (with the highest mortality rate).

On exclusion of the 2 institutions that reported no operative mortality, in the remaining set of patients $(27,174)$, the best accuracy was obtained by the ACEF score (AUC 0.74), followed by the logistic euroSCORE and the additive euroSCORE. In this subset, the odds ratio for the ACEF score was 2.76 (95\% CI, 2.52-3.01), with an estimated operative mortality increase of $102 \%$ per each ACEF score point.

The predictive accuracy of the ACEF score was tested using a 10-fold cross-validation (Table 3), separately for the whole population and the subset of patients obtained excluding the institutions without operative mortality. The robustness of the ACEF score was good in both settings (overall population: mean $\mathrm{AUC}_{\mathrm{cv}}=0.699$ vs mean $\mathrm{AUC}_{\text {one-shot }}=0.701$; subset of institutions reporting operative mortality: mean $\mathrm{AUC}_{\mathrm{cv}}=0.737$ vs mean $\mathrm{AUC}_{\text {one-shot }}=0.74$ ).

The clinical performance of the risk models is shown in Figure 2. The patient population was divided in deciles according to the logistic euroSCORE mortality risk. For each decile, the observed operative mortality rate (with 95\% CI) and predicted mortality rate (with $95 \% \mathrm{CI}$ ) were calculated, according to the additive euroSCORE, logistic euroSCORE, and ACEF.

Both the additive and logistic euroSCOREs significantly overestimated the operative mortality risk in all deciles. The ACEF score significantly overestimated the operative mortality risk in the first decile (observed mortality rate, $0.3 \%$; $95 \% \mathrm{CI}, 0.1-0.6$ vs predicted mortality rate, $1.5 ; 95 \% \mathrm{CI}$, $1.47-1.51$ ) and the second decile (observed mortality rate, $0.8 \% ; 95 \%$ CI, $0.5-1.1$ vs predicted mortality rate, 1.8 ; $95 \%$ CI, 1.76-1.83). The ACEF score correctly estimated

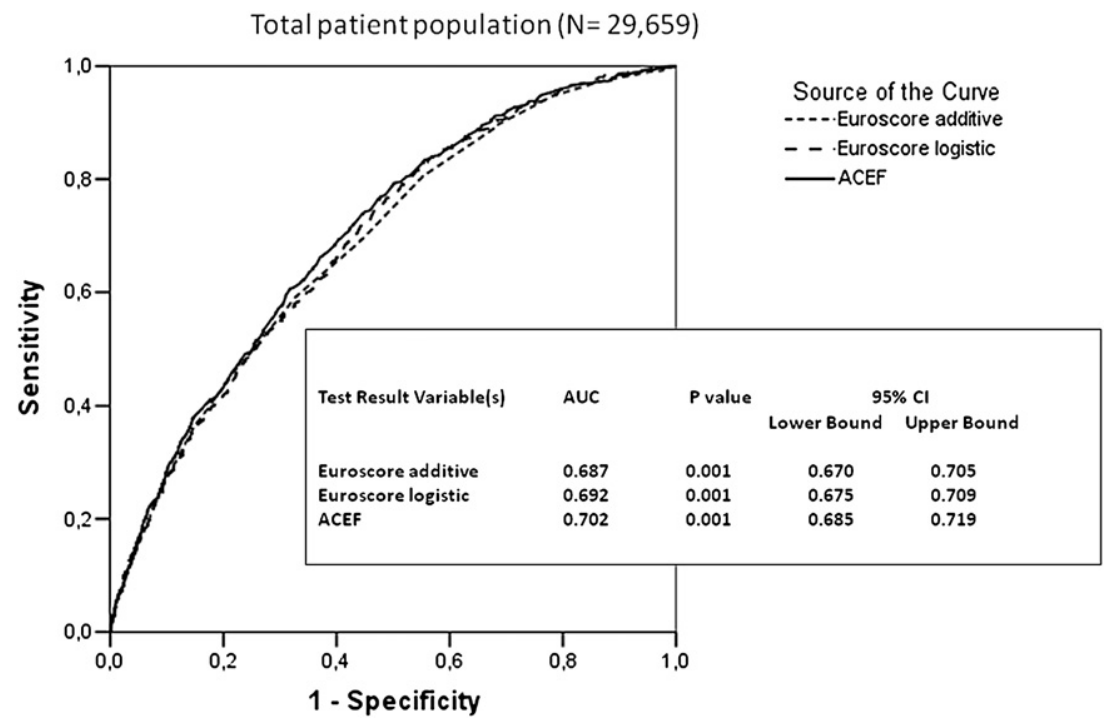

FIGURE 1. Receiver operating characteristics analysis for the 3 models. $A C E F$, Age, creatinine, ejection fraction; $A U C$, area under the curve; $C I$, confidence interval. 
TABLE 2. Accuracy of the predictive models in the different participating institutions

\begin{tabular}{lccccc}
\hline & $\begin{array}{c}\text { Patients } \\
\text { Institution }\end{array}$ & $\begin{array}{c}\text { Operative } \\
\text { mortality (\%) }\end{array}$ & $\begin{array}{c}\text { ACEF score } \\
\text { AUC (95\% CI) }\end{array}$ & $\begin{array}{c}\text { euroSCORE (additive) } \\
\text { AUC (95\% CI) }\end{array}$ & $\begin{array}{c}\text { euroSCORE (logistic) } \\
\text { AUC (95\% CI) }\end{array}$ \\
\hline 1 & 727 & 0 & Not applicable & Not applicable & Not applicable \\
2 & 1,758 & 0 & Not applicable & Not applicable & Not applicable \\
3 & 821 & 2.43 & $0.78(0.67-0.89)$ & $0.77(0.67-0.86)$ & $0.77(0.67-0.87)$ \\
4 & 18,998 & 3.49 & $0.74(0.71-0.75)$ & $0.69(0.67-0.71)$ & $0.69(0.67-0.71)$ \\
5 & 307 & 5.86 & $0.57(0.46-0.68)$ & $0.62(0.51-0.74)$ & $0.61(0.49-0.72)$ \\
6 & 6,432 & 1.66 & $0.78(0.74-0.82)$ & $0.75(0.71-0.79)$ & $0.73(0.68-0.77)$ \\
7 & 616 & 2.44 & $0.78(0.65-0.91)$ & $0.83(0.73-0.93)$ & $0.82(0.72-0.93)$ \\
All institutions & 29,659 & 2.77 & $0.70(0.68-0.72)$ & $0.68(0.66-0.70)$ & $0.69(0.67-0.71)$ \\
Excluding institutions 1 and 2 & 27,174 & 3.02 & $0.74(0.72-0.76)$ & $0.70(0.68-0.71)$ & $0.71(0.69-0.73)$ \\
\hline
\end{tabular}

the mortality risk in deciles 3 to 9 (observed mortality range, $1.4 \%-4.1 \%$ ). The ACEF score significantly underestimated the operative mortality risk in the 10th decile (observed mortality rate, $7.2 \%$; 95\% CI, $6.2-8.1$ vs predicted mortality rate, $5.52 ; 95 \% \mathrm{CI}, 5.27-5.77)$.

\section{DISCUSSION}

Mortality after cardiac surgery is still a relevant issue, and a clear definition of this event is still lacking. The concept of "operative mortality" is based on in-hospital mortality or outside-hospital mortality occurring within 30

TABLE 3. Ten-fold cross-validation of the age, creatinine, ejection fraction score

\begin{tabular}{lccccc}
\hline \multicolumn{5}{c}{ Overall population } \\
\hline Partition & Frequency & Percent & $\begin{array}{c}\text { Cumulative } \\
\text { frequency }\end{array}$ & $\begin{array}{c}\text { Cumulative } \\
\text { percent }\end{array}$ & AUC \\
\hline 0 & 2965 & 10.00 & 2965 & 10.00 & 0.721 \\
1 & 2966 & 10.00 & 5931 & 20.00 & 0.694 \\
2 & 2966 & 10.00 & 8897 & 30.00 & 0.695 \\
3 & 2966 & 10.00 & 11,863 & 40.00 & 0.688 \\
4 & 2966 & 10.00 & 14,829 & 50.00 & 0.702 \\
5 & 2966 & 10.00 & 17,795 & 60.00 & 0.687 \\
6 & 2966 & 10.00 & 20,761 & 70.00 & 0.693 \\
7 & 2966 & 10.00 & 23,727 & 80.00 & 0.701 \\
8 & 2966 & 10.00 & 26,693 & 90.00 & 0.707 \\
9 & 2966 & 10.00 & 29,659 & 100.00 & 0.705 \\
\hline
\end{tabular}

Excluding institutions without operative mortality

\begin{tabular}{|c|c|c|c|c|c|}
\hline Partition & Frequency & Percent & $\begin{array}{l}\text { Cumulative } \\
\text { frequency }\end{array}$ & $\begin{array}{c}\text { Cumulative } \\
\text { percent }\end{array}$ & AUC \\
\hline 0 & 2717 & 10.00 & 2717 & 10.00 & 0.717 \\
\hline 1 & 2717 & 10.00 & 5434 & 20.00 & 0.741 \\
\hline 2 & 2718 & 10.00 & 8152 & 30.00 & 0.726 \\
\hline 3 & 2717 & 10.00 & 10,869 & 40.00 & 0.717 \\
\hline 4 & 2718 & 10.00 & 13,587 & 50.00 & 0.779 \\
\hline 5 & 2717 & 10.00 & 16,304 & 60.00 & 0.742 \\
\hline 6 & 2718 & 10.00 & 19,022 & 70.00 & 0.766 \\
\hline 7 & 2717 & 10.00 & 21,739 & 80.00 & 0.698 \\
\hline 8 & 2718 & 10.00 & 24,457 & 90.00 & 0.737 \\
\hline 9 & 2717 & 10.00 & 27,174 & 100.00 & 0.747 \\
\hline
\end{tabular}

days from the operation. This outcome measurement was considered by the only 3 risk scores that are presently accepted (class I for euroSCORE and STS score, class IIb for ACEF score) by the joint European Society of Cardiology-European Association of Cardiac and Thoracic Surgery Guidelines for Myocardial Revascularization. ${ }^{5}$ However, it should be considered that this interval is arbitrary, and that cardiac surgery-related mortality may extend far beyond 30 days from the operation. More accurate risk models with a different time-dependent outcome measurement are suggested, but so far we can address this relevant point using the existing tools.

The overall accuracy of the ACEF score in this external validation series (AUC 0.7) is lower than in the internal validation series (AUC 0.8). ${ }^{1}$ However, 2 institutions contributed a series of patients unsuitable for risk score validation (no events), and when excluding these institutions the accuracy increased to an acceptable AUC of 0.74 . The ACEF score (and the euroSCORE) showed poor performance in only 1 institution, which had the highest mortality rate in our series.

According to our findings, the ACEF score performs better than the widespread and established euroSCORE in patients undergoing elective cardiac surgery. In particular, we confirmed the results of the first study introducing the ACEF score ${ }^{1}$ in a larger patient population collected at multiple sites. In this series, the ACEF score has a noninferior accuracy with respect to the additive and logistic euroSCOREs and a better clinical performance in elective patients. Despite being based on only 3 risk factors, the ACEF score offers a level of accuracy comparable to more complex scores based on more than 10 risk factors. There are many possible explanations for this apparent paradox, which have been widely addressed in a previous study ${ }^{1}$; however, it may be useful to remember that when numerous variables are included in an attempt to "control" or "adjust" the data, the accuracy of the results may be threatened, ${ }^{6}$ and the general advice of statisticians is to be parsimonious in selecting independent variables. ${ }^{7}$ This concept was recently ${ }^{8}$ applied to the euroSCORE, with 


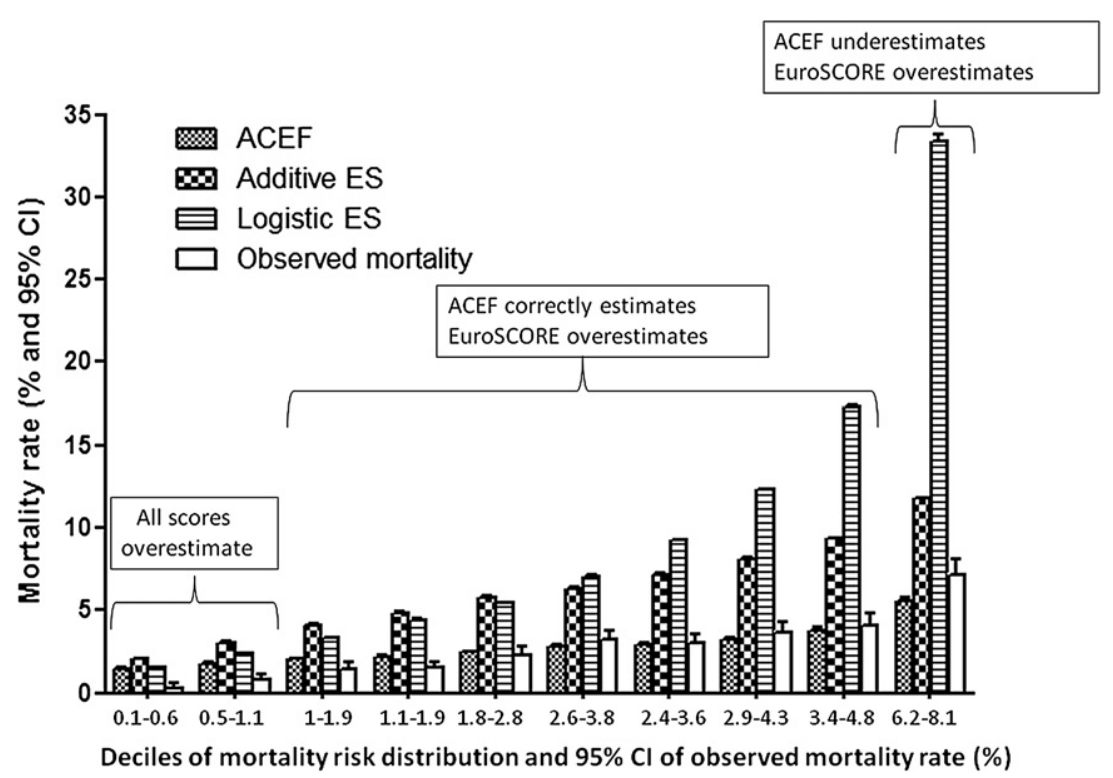

FIGURE 2. Predicted versus observed operative mortality rates for deciles of risk distribution. ACEF, Age, creatinine, ejection fraction; $C I$, confidence interval; ES, European System for Cardiac Operative Risk Evaluation.

a demonstration that the statistical accuracy of this model does not increase if other factors are added to the first 5 most important risk factors: age, $\mathrm{EF}$, preoperative creatinine, emergency operation, and non-isolated coronary operation. Conversely, there is a reduction in the related calibration properties.

Our data confirm the well-known problem of operative mortality risk overestimation attributable to the euroSCORE. There was a 2- to 3 -fold difference between the predicted and the observed mortality when the prediction was based on the additive and logistic euroSCOREs, respectively. The existence and magnitude of this discrepancy have been highlighted in other studies. ${ }^{9-15}$ The euroSCORE significantly overestimated the operative mortality risk in every subset of risk, with the highest discrepancy in the highest risk decile, whereas the logistic euroSCORE predicted a mortality 5 -fold higher than that observed.

The mortality rate predicted by the ACEF score was not significantly different from that observed for the entire population (with a negligible difference of $0.07 \%$ ). Conversely, there was a significant overestimation in the 2 lowest deciles of risk distribution, with a predicted mortality rate less than $2 \%$ and an observed mortality rate less than $1 \%$. Although significant, this difference is not important in clinical terms. In very low-risk patients, the assumption that the predictable mortality is in the range of $1 \%$ to $2 \%$ may be accepted, and subtle differences in this area are difficult to detect.

In the third to ninth deciles of risk distribution, the predicted mortality rate obtained by the ACEF score was not significantly different from the observed mortality rate. This observation pertains to an observed mortality risk ranging from $1.4 \%$ to $4.1 \%$, which is most representative for a population of adult patients undergoing elective cardiac surgery.

In contrast with the euroSCORE, the ACEF score significantly underestimated the operative mortality risk in patients at high risk (10th decile, observed mortality rate $7.2 \%$ ), with a predicted risk of $5.5 \%$. Even if this difference may be considered acceptable in clinical terms, some hypotheses derive from this pattern. First, 3 factors are probably too few to predict the operative mortality in very high-risk patients. A previous study ${ }^{8}$ demonstrated that when the observed mortality rate is approximately $7 \%$, adequate predictive models should include 7 to 12 risk factors. Second, there are issues related to the "extreme" risk conditions. Some of the elective patients who do not survive the operation actually die as a consequence of 1 or more of these conditions, which may include severe chronic obstructive pulmonary disease under oxygen therapy, morbid obesity (body mass index $>40$ ), neurologic impairment severely limiting the patient mobility, frailty, congenital prothrombotic or prohemorrhagic disorders, and many others. None of these conditions is included in any of the existing risk scores, and the ACEF score is not an exception.

The ACEF score offers some advantages with respect to the other existing risk scores. It is simple and based on numbers, not clinical conditions that may suffer from individual interpretation and consequent operator-related biases. The left ventricular EF is included as a continuous variable to account for the extremely high risk attributable to patients with $\mathrm{EF}$ in the range of $10 \%$ to $30 \%$. In this area, the risk increases exponentially with decreasing EF. The ACEF score does not pool together all the patients with an EF 
less than $30 \%$, as do the other risk scores. As increasingly more patients with poor left ventricular EF are undergoing cardiac operations, this feature of the ACEF score may be of considerable importance.

A distinctive pattern of the ACEF score is that it is not a broad measure and instead focuses exclusively on patients undergoing elective cardiac surgery. The ACEF score does not aim to predict risk in emergency clinical scenarios. This may be seen as a limitation; however, the majority of the patients undergoing cardiac surgery are elective cases; moreover, excluding critical scenarios is probably one of the reasons for the improved clinical performance.

The most important finding of our study is that, in any case, both the ACEF score and the euroSCORE failed to reach optimal levels of accuracy (AUC $>0.8$ ). Until the problem of predicting mortality after cardiac surgery is addressed with the usual statistical approaches, it will always be difficult to achieve this goal, regardless of the use of parsimonious or complex models. The existence of rare, but potentially lethal risk factors that are usually excluded by logistic models is one of the main problems when trying to individualize the risk prediction, and certainly many high-risk patients may not receive a correct evaluation with the existing risk models.

A limitation of the present study is that we compared the ACEF score with the well-established additive and logistic euroSCOREs, but we could not include the STS-predicted risk of mortality (PROM) score in the comparison. The STS-PROM is a well-performing model that includes a considerable number of risk factors, with different calculations based on the type of operation. This algorithm underlying the STS-PROM score is not available, and therefore retrospective calculations are impossible.

\section{CONCLUSIONS}

Our study confirms that a simple risk score may offer the same accuracy and better clinical performance than more complex scores in the setting of patients undergoing elective cardiac surgery. The ACEF score may be a good starting point for further studies in the field of risk stratification in cardiac surgery and should be considered as a simple "skeleton" to build up different models that apply to different clinical scenarios, which is actually what the cardiologists did when they integrated the ACEF and Syntax scores. ${ }^{2}$ The previously mentioned "extreme" risk conditions that are usually left outside the risk scores because of their low prevalence may be tested one by one or in combination to increase the performance of the ACEF score in the area of high-risk operations. Further improvements of the ACEF score could be based on statistical techniques that differ from the usual logistic regression analysis and test potential additional factors to increase the "risk stratification capacity" for the detection of specific high-risk patient populations (eg, patient selection for transcatheter aortic valve implantation) with risk-stratification tables. ${ }^{16,17}$ The present validation study justifies the inclusion of the ACEF score within the existing risk stratification scores in cardiac surgery, ${ }^{5}$ opening the door to possible further improvements of this model.

The authors thank Dr Maria Cristina Barattoni, Dr Luca Ghezzi (Villa Maria Foundation-GMC Care and Research), and Valeria Pistuddi (IRCCS Policlinico S. Donato) for contributions with regard to data extraction and analysis.

\section{References}

1. Ranucci M, Castelvecchio S, Menicanti L, Frigiola A, Pelissero G. The risk of assessing mortality risk in elective cardiac operations. Age, creatinine, ejection fraction and the law of parsimony. Circulation. 2009;119:3053-61.

2. Garg S, Sarno G, Garcia-Garcia HM, Girasis C, Wykrzykowska J, Dawkins KD, et al. A new tool for the risk stratification of patients with complex coronary artery disease: the Clinical Syntax score. Circ Cardiovasc Interv. 2010;3:317-26.

3. Roques F, Nashef SA, Michel P, Gauducheau E, de Vincentiis C, Baudet E, et al. Risk factors and outcome in European cardiac surgery: analysis of the EuroSCORE multinational database of 19030 patients. Eur J Cardiothorac Surg. 1999; 15:816-22.

4. Roques F, Michel P, Goldstone AR, Nashef SA. The logistic EuroSCORE. Eur Heart J. 2003;24:881-2.

5. Wijns W, Kolh P, Danchin N, Di Mario C, Falk V, Folliguet T, et al. Guidelines on myocardial revascularization: the Task Force on Myocardial Revascularization of the European Society of Cardiology (ESC) and the European Association for Cardio-Thoracic Surgery (EACTS). Eur Heart J. 2010;31:2501-55. Epub 2010 Aug 29.

6. Concato J, Feinstein AR, Holford T. The risk of determining risk with multivariable models. Ann Intern Med. 1993;118:201-10.

7. Wells CK, Feinstein AR, Walter SD. A comparison of multivariable mathematical methods for predicting survival-III. Accuracy of predictions in generating and challenge sets. J Clin Epidemiol. 1990;43:361-72.

8. Ranucci M, Castelvecchio S, Menicanti L, Frigiola A, Pelissero G. Accuracy, calibration, and clinical performance of the EuroSCORE: can we reduce the number of variables? Eur J Cardiothorac Surg. 2010;37:724-9.

9. Yap CH, Reid C, Yii M, Rowland MA, Mohajeri M, Skillington PD, et al. Validation of the EuroSCORE model in Australia. Eur J Cardiothorac Surg. 2006;29: 441-6.

10. Jin R, Grunkemeier GL, Providence Health System Cardiovascular Study Group. Does the logistic EuroSCORE offer an advantage over the additive model? Interact Cardiovasc Thorac Surg. 2006;5:15-7.

11. Shanmugam G, West M, Berg G. Additive and logistic EuroSCORE performance in high risk patients. Interact Cardiovasc Thorac Surg. 2005;4:299-303.

12. Bhatti F, Grayson AD, Grotte G, Fabri BM, Au J, Jones M, et al. North West Quality Improvement Programme in Cardiac Interventions. The logistic EuroSCORE in cardiac surgery: how well does it predict operative risk? Heart. 2006;92:1817-20.

13. Zingone B, Pappalardo A, Dreas L. Logistic versus additive EuroSCORE. A comparative assessment of the two models in an independent population sample. Eur J Cardiothorac Surg. 2004;26:1134-40.

14. Dewey TM, Brown D, Ryan WH, Herbert MA, Prince SL, Mack MJ. Reliability of risk algorithms in predicting early and late operative outcomes in high-risk patients undergoing aortic valve replacement. J Thorac Cardiovasc Surg. 2008;135: 180-7.

15. Ranucci M, Castelvecchio S, Menicanti LA, Scolletta S, Biagioli B, Giomarelli P. An adjusted EuroSCORE model for high-risk cardiac patients. Eur J Cardiothorac Surg. 2009;36:791-7.

16. Cook NR. Use and misuse of the receiver operating characteristics curve in risk prediction. Circulation. 2007;115:928-35.

17. Cook NR, Buring JE, Ridker PM. The effect of including C-reactive protein in cardiovascular risk prediction models for women. Ann Intern Med. 2006; 145:21-9. 